\title{
A Cross-Layer Design for Generic Interference-Limited Multicarrier Networks
}

\author{
Rozita Rashtchi, Ramy H. Gohary, and Halim Yanikomeroglu \\ Department of System and Computer Engineering, Carleton University, Ottawa, Ontario, Canada \\ \{rozita, gohary, halim\}@sce.carleton.ca
}

\begin{abstract}
In this paper, we develop a novel framework for optimizing data routes, subcarrier schedules and power allocations in generic half-duplex interference-limited multicarrier networks. In this framework each subcarrier can be both reused and time-shared by multiple nodes. Thus, this framework subsumes designs in which time-sharing and frequency-reuse are considered separately, and can therefore offer significant performance gains over them. Considering both time-sharing and frequency-reuse jointly gives rise to generally difficult to solve non-convex optimization problems. However, using approximation techniques based on geometric programming, we provide a computationallyefficient method for obtaining locally optimal solutions. Numerical examples confirm that the framework proposed herein yields achievable data rates that are superior to those yielded by currently available designs.
\end{abstract}

Index Terms-Frequency-reuse, geometric programming, power allocation, routing, scheduling, time-sharing.

\section{INTRODUCTION}

To meet prospective demands for high data rate services, future wireless networks must judiciously exploit the scarce resources available for them. These resources include time, frequency and the typically low power batteries. To exploit these resources, network functionalities, e.g., routing, scheduling and power allocation, must be designed jointly across multiple network layers to avoid the performance degradation that can result from designing these functionalities separately [1].

Cross-layer designs can be classified according to whether the available subcarriers can be reused by multiple nodes simultaneously and whether these subcarriers can be timeshared by them. Although using a subcarrier exclusively by one node (i.e., without reuse) throughout the signalling interval (i.e., without time-sharing) is relatively easy to implement in practice, this approach limits the network capabilities and deprives it from achieving rates that can be reliably communicated over it. Developing optimization frameworks that allow the subcarriers to be reused and time-shared jointly will enhance the network capabilities and will enable it to support data rates well beyond those supported by networks that consider frequency-reuse and time-sharing separately [2].

Cross-layer designs in which frequency-reuse is not allowed and the subcarriers are restricted to be used by only one node throughout the signalling interval give rise to NP-hard binary scheduling problems [2], which can be solved suboptimally using the algorithms in [3]-[6]. For networks in which

This work is supported in part by the Natural Sciences and Engineering Research Council of Canada (NSERC). frequency-reuse is not allowed but the subcarriers are timeshared, several cross-layer designs can be cast in a convex form; see e.g., [6]-[8]. Including frequency-reuse renders the network interference-limited. This case without time-sharing has been considered in [9] and [10] for single-carrier networks and in [11] for multicarrier ones.

Allowing subcarriers to be both time-shared and frequencyreused by multiple nodes conforms to the emerging Long Term Evolution (LTE) standard [12] and is the main focus of this paper. To the best of our knowledge, this framework has never been considered in the literature and subsumes currently available ones as special cases. We consider a generic half-duplex multicarrier network in which nodes can be sources, destinations and/or relays. We develop a cross-layer optimization framework that incorporates data routing, subcarrier scheduling and power allocation to maximize a weighted sum of the rates injected and reliably communicated over the network. Considering both time-shared scheduling and frequency-reuse in this framework gives rise to a non-convex cross-layer design which is generally difficult to solve. However, using approximation techniques based on geometric programming (GP), we provide a computationally-efficient method for obtaining locally optimal solutions. In particular, in this method the constraints that are not GP-compatible are approximated with monomial expressions around an initial point. An iterative technique, known as the single-condensation method, is then used to obtain a feasible solution that satisfies the KarushKuhn-Tucker (KKT) conditions. Numerical examples confirm that the framework proposed herein yields achievable data rates that are superior to those yielded by currently available designs. Finally, we note that the optimization framework developed herein is centralized and, in practice, is solved for every scheduling interval which contains multiple OFDM symbols. Thus, this framework is suitable for designing small to medium size networks. For larger networks, this framework can be seen as a benchmark and a first step towards developing effective distributed designs that make judicious use of the degrees of freedom offered by the physical wireless medium.

\section{OPTIMIZATION FRAMEWORK}

Consider an OFDMA-based network with $K$ subcarriers, $N$ nodes and $L$ links. Let $\mathcal{K} \triangleq\{1, \ldots, K\}, \mathcal{N} \triangleq\{1, \ldots, N\}$ and $\mathcal{L} \triangleq\{1, \ldots, L\}$ be the sets of subcarriers, nodes and links in the network, respectively. To facilitate the enumeration of the $L=N(N-1)$ links in the network, the link from node $n$ to 
node $n^{\prime}$ will be labelled by $\ell=(N-1)(n-1)+n^{\prime}-1$ if $n<n^{\prime}$ and by $\ell=(N-1)(n-1)+n^{\prime}$ if $n>n^{\prime}$. Let $\mathcal{O}(n)$ and $\mathcal{I}(n)$ be the sets of links outgoing from and incoming to node $n$, respectively. The connectivity of network is captured by the incidence matrix, $A$, with entries $a_{n \ell}=1$ if link $\ell \in \mathcal{O}(n)$, $a_{n \ell}=-1$ if link $\ell \in \mathcal{I}(n)$, and $a_{n \ell}=0$ otherwise.

Each node has one transmit and one receive antenna, and a power budget, $P_{n}, n \in \mathcal{N}$. Each node is capable of sending, receiving and relaying data to other nodes. An instance of such a network with $N=3$ and $K=4$ is depicted in Figure 1.

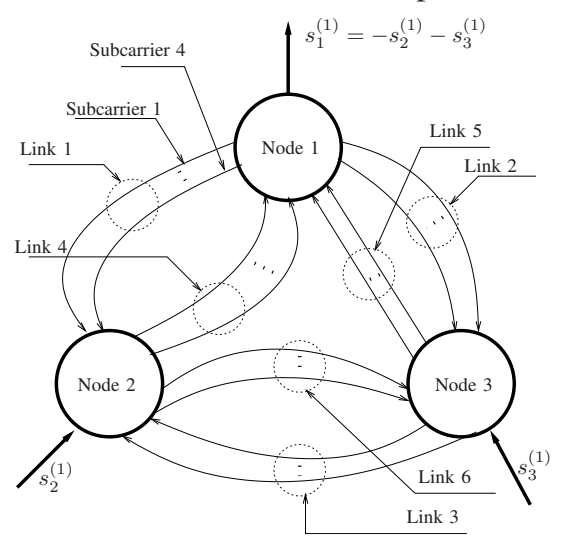

Fig. 1: A network with $N=3, K=4$ and $D=\{1\}$.

The channel complex coefficient on subcarrier $k \in \mathcal{K}$ of link $\ell \in \mathcal{L}$ is denoted by $h_{\ell k}$, and is assumed to remain constant during the signalling interval. For practical considerations, we assume half-duplex operation, i.e., a node cannot send and receive data on the same subcarrier at the same time.

\section{A. System Objective}

Let $\mathcal{D}$ be the set of all destination nodes, $\mathcal{D} \subseteq \mathcal{N}$. Let $s_{n}^{(d)}$ be the rate of data injected into the network at node $n$ and intended for destination $d$. For each $s_{n}^{(d)}$, we assign a predetermined weight, $w_{n}^{(d)}$. Our objective is to maximize the weighted sum of the rates injected into the network, i.e.,

$$
\max \sum_{d \in \mathcal{D}} \sum_{n \in \mathcal{N} \backslash\{d\}} w_{n}^{(d)} s_{n}^{(d)},
$$

where \is the set minus operation. Assigning weights to injected rates not only controls the quality of service offered to each destination, but also provides the region of the rates that can be achieved by the proposed design.

\section{B. System Constraints}

In this section we provide mathematical formulations for the constraints that must be satisfied by the network variables.

1) Routing Constraints: Let $x_{\ell k}^{(d)}$ be the rate of the data intended for destination $d$ on subcarrier $k$ of link $\ell$. These variables will be responsible for determining the route taken by each data stream. To ensure continuous routes from sources to destinations, the flow conservation law must be satisfied at each node. Using the incidence matrix $A$ in Section II, this law can be represented as

$$
\sum_{\ell \in \mathcal{L}} \sum_{k \in \mathcal{K}} a_{n \ell} x_{\ell k}^{(d)}=s_{n}^{(d)}, \quad n \in \mathcal{N} \backslash\{d\}, d \in \mathcal{D},
$$

where $\left\{x_{\ell k}^{(d)}\right\}$ and $\left\{s_{n}^{(d)}\right\}$ are non-negative, i.e.,

$$
\begin{array}{ll}
x_{\ell k}^{(d)} \geq 0, & d \in \mathcal{D}, \ell \in \mathcal{L}, k \in \mathcal{K}, \\
s_{n}^{(d)} \geq 0, & d \in \mathcal{D}, n \in \mathcal{N} \backslash\{d\} .
\end{array}
$$

2) Scheduling Constraints: Considering both time-sharing and frequency-reuse simultaneously requires us to introduce a set of new variables to refer to the links that operate simultaneously on the same subcarrier for a fraction of the signalling interval. To do so, we let $\gamma_{\ell}^{(k)}$ be the variable that determines the fraction of the signalling interval during which only link $\ell \in \mathcal{L}$ is 'active' on subcarrier $k \in \mathcal{K}$ and all other links are 'silent' on the same subcarrier. Also let $\gamma_{\ell \ell^{\prime}}^{(k)}$ be a variable that determines the fraction of the signalling interval during which both links $\ell$ and $\ell^{\prime} \in \mathcal{L}, \ell \neq \ell^{\prime}$ are active on subcarrier $k \in \mathcal{K}$ but all other links are silent. Similarly, $\gamma_{\ell \ell^{\prime} \ell^{\prime \prime}}^{(k)}$ represents the fraction of the signalling interval that distinct links $\ell, \ell^{\prime}$ and $\ell^{\prime \prime} \in \mathcal{L}$ are active on subcarrier $k \in \mathcal{K}$ and all other links are silent. Then $\gamma_{12 \cdots L}^{(k)}$ is the fraction of the signalling interval that all links are active on subcarrier $k \in \mathcal{K}$. Note that the indices are interchangeable, i.e, $\gamma_{\ell \ell^{\prime}}^{(k)}$ is equivalent to $\gamma_{\ell^{\prime} \ell}^{(k)}$. Therefore for consistency, we write the indices in an ascending order. For example, we will use $\gamma_{12}^{(k)}$ for both $\gamma_{12}^{(k)}$ and $\gamma_{21}^{(k)}$. Let $\Gamma$ be the set containing all the subcarrier timeshares described above. For feasible subcarrier scheduling, all the elements in $\Gamma$ should be nonnegative, i.e.,

$$
\Gamma \geq 0, \quad \text { elementwise. }
$$

Remark: In general, the cardinality of $\Gamma,|\Gamma|$, increases exponentially with the number of nodes, $N$. However, for large networks, one can limit the number of links that contribute to simultaneous transmissions. This limitation decreases $|\Gamma|$ and can be readily incorporated in the formulation.

To avoid overlapping in time, the total amount of time that subcarrier $k \in \mathcal{K}$ is used cannot exceed the length of the signalling interval. As such,

$$
\sum_{\ell \in \mathcal{L}} \gamma_{\ell}^{(k)}+\sum_{\ell \in \mathcal{L}} \sum_{\ell^{\prime} \in \mathcal{L} \backslash\{\ell\}} \gamma_{\ell \ell^{\prime}}^{(k)}+\cdots+\gamma_{12 \cdots L}^{(k)} \leq 1, \quad \forall k \in \mathcal{K} .
$$

In order to realize the practical half-duplex assumption, we must ensure that the nodes do not transmit and receive data on one subcarrier at the same time. In other words, let $\ell \in$ $\mathcal{I}(n)$ and $\ell^{\prime} \in \mathcal{O}(n)$. From the half-duplex assumption, these two links cannot be active on subcarrier $k$ at the same time. Therefore all the time-shares that include $\ell$ and $\ell^{\prime}$, must be zero. This constraints can be written as

$$
\begin{array}{r}
a_{n \ell}^{+} a_{n \ell^{\prime}}^{+}\left(\gamma_{\ell \ell^{\prime}}^{(k)}+\sum_{\ell^{\prime \prime} \in \mathcal{L} \backslash\left\{\ell, \ell^{\prime}\right\}} \gamma_{\ell \ell^{\prime} \ell^{\prime \prime}}^{(k)}+\ldots+\gamma_{12 \cdots L}^{(k)}\right)=0, \\
\ell \in \mathcal{L}, \ell^{\prime} \in \mathcal{L} \backslash\{\ell\}, k \in \mathcal{K},
\end{array}
$$

where $a_{n \ell}^{+}=\max \left\{0, a_{n \ell}\right\}$, that is, $a_{n \ell}^{+}=1$ if $\ell \in \mathcal{O}(n)$ and zero, otherwise. It is also assumed that nodes are not able to broadcast data to different destinations at the same time. More specifically, at any time instance, node $n$ can have at most one active link on each subcarrier. This can be represented as 


$$
\begin{array}{r}
a_{n \ell}^{+} a_{n \ell^{\prime}}^{-}\left(\gamma_{\ell \ell^{\prime}}^{(k)}+\sum_{\ell^{\prime \prime} \in \mathcal{L} \backslash\left\{\ell, \ell^{\prime}\right\}} \gamma_{\ell \ell^{\prime} \ell^{\prime \prime}}^{(k)}+\ldots+\gamma_{12 \cdots L}^{(k)}\right)=0, \\
\ell \in \mathcal{L}, \ell^{\prime} \in \mathcal{L} \backslash\{\ell\}, k \in \mathcal{K},
\end{array}
$$

where $a_{n \ell}^{-}=\min \left\{0, a_{n \ell}\right\}$, that is, $a_{n \ell}^{-}=-1$ if $\ell \in \mathcal{I}(n)$ and zero, otherwise. Note that (7) and (8) take effect only when $a_{n \ell}^{+} a_{n \ell^{\prime}}^{+} \neq 0$ and $a_{n \ell}^{+} a_{n \ell^{\prime}}^{-} \neq 0$, respectively.

3) Power Allocation Constraints: Let $p_{\ell k}$ be the power allocated for transmission on subcarrier $k$ of link $\ell$. The power emitted by each node on scheduled outgoing links must be non-negative and must satisfy the total power budget constraint. Hence,

$$
p_{\ell k} \geq 0, \quad \ell \in \mathcal{L}, k \in \mathcal{K},
$$

and

$\sum_{k \in \mathcal{K}} \sum_{\ell \in \mathcal{O}(n)}\left(\gamma_{\ell}+\gamma_{\ell \ell^{\prime}}^{(k)}+\sum_{\ell^{\prime \prime} \in \mathcal{L} \backslash\left\{\ell, \ell^{\prime}\right\}} \gamma_{\ell \ell^{\prime} \ell^{\prime \prime}}^{(k)}+\ldots+\gamma_{12 \cdots L}^{(k)}\right) p_{\ell k} \leq P_{n}$ $n \in \mathcal{N}$

4) Capacity Constraints: For each link $\ell \in \mathcal{L}$ and subcarrier $k \in \mathcal{K}$, the capacity constraint can be written as

$$
\begin{aligned}
\sum_{d \in \mathcal{D}} x_{\ell k}^{(d)} \leq & \gamma_{\ell}^{(k)} \log \left(1+p_{\ell k} g_{\ell k}\right) \\
& +\sum_{\ell^{\prime} \in \mathcal{L} \backslash\{\ell\}} \gamma_{\ell \ell^{\prime}}^{(k)} \log \left(1+\frac{p_{\ell k} g_{\ell k}}{1+p_{\ell^{\prime} k} g_{\ell^{\prime \prime} k}}\right) \\
& \vdots \\
+ & \gamma_{12 \cdots L}^{(k)} \log \left(1+\frac{p_{\ell k} g_{\ell k}}{1+\sum_{\ell^{\prime} \in \mathcal{L} \backslash\{\ell\}} p_{\ell^{\prime} k} g_{\ell^{\prime \prime} k}}\right)
\end{aligned}
$$

where $g_{\ell k} \triangleq \frac{\left|h_{\ell k}\right|^{2}}{\sigma^{2}}$ and $\sigma^{2}$ is the power of the additive Gaussian noise at the receiver. In this constraint, $\ell^{\prime \prime}$ is used to denote the index of the link connecting the node at which link $\ell^{\prime}$ originates to the node at which link $\ell$ ends. The first term on the right-hand-side (RHS) of (11) is related to a fraction of time that only link $\ell$ is active on subcarrier $k$, and the second term is related to a fraction of time in which only two links $\ell$ and $\ell^{\prime} \in \mathcal{L} \backslash\{\ell\}$ are active on subcarrier $k$, and so on.

\section{Problem Formulation}

Combining the objective in (1) with the constraints in (2)(11), yields a formulation for the cross-layer design in interference-limited multicarrier networks with time-shared subcarriers. In particular, this formulation can be cast as

$$
\max _{\left\{s_{n}^{(d)}\right\},\left\{x_{\ell k}^{(d)}\right\},\left\{p_{\ell k}\right\}, \Gamma} \sum_{d \in \mathcal{D}} \sum_{n \in \mathcal{N} \backslash\{d\}} w_{n}^{(d)} s_{n}^{(d)},
$$

subject to Routing constraints in (2)-(4),

Power allocation constraints in (9)-(10), Scheduling constraints in (5)-(8),

$$
\text { Capacity constraints in (11). }
$$

The optimization problem in (12) is highly nonconvex because of the scheduling constraints in (7) and (8), the power allocation constraints in (10) and the capacity constraints in (11).
To alleviate this difficulty, in the next section, we will develop a GP-based algorithm to obtain a locally optimal solution for this problem.

\section{Proposed GP-BAsed Algorithm}

The optimization problem in (12), although nonconvex, shares some common features with the GP framework [13]. In order to take the advantage of this framework, we will define two new sets of variables, $\left\{t_{n}^{(d)}\right\}$ and $\left\{r_{\ell k}^{(d)}\right\}$, which are related to $\left\{s_{n}^{(d)}\right\}$ and $\left\{x_{\ell k}^{(d)}\right\}$ by the following bijective maps:

$$
\begin{aligned}
s_{n}^{(d)}=\log _{2} t_{n}^{(d)}, & & n \in \mathcal{N} \backslash\{d\}, d \in \mathcal{D}, \\
x_{\ell k}^{(d)}=\log _{2} r_{\ell k}^{(d)}, & & \ell \in \mathcal{L}, k \in \mathcal{K}, d \in \mathcal{D} .
\end{aligned}
$$

Using the new variables, the routing constraints in Section II-B1, can be expressed in GP-compatible forms, i.e,

$$
\begin{array}{rlrl}
\prod_{\ell \in \mathcal{L}} \prod_{k \in \mathcal{K}}\left(r_{\ell k}^{(d)}\right)^{a_{n \ell}} & =t_{n}^{(d)}, & & n \in \mathcal{N} \backslash\{d\}, d \in \mathcal{D}, \\
r_{\ell k}^{(d)} \geq 1, & & \ell \in \mathcal{L}, k \in \mathcal{K}, d \in \mathcal{D}, \\
t_{n}^{(d)} \geq 1, & & n \in \mathcal{N} \backslash\{d\}, d \in \mathcal{D} .
\end{array}
$$

The non-negativity constraints in (9) and (5) are inherently satisfied in the GP framework. The constraints in (6) and (10) are already in a GP-compatible form, because their left-handsides (LHSs) are posynomials and their RHSs are monomials. Because the RHSs of (7) and (8) are equal to zero, these constraints do not conform to the GP-standard framework. To alleviate this difficulty, we replace these equality constraints with the following inequality ones:

$$
\begin{gathered}
a_{n \ell}^{+} a_{n \ell^{\prime}}^{+}\left(\gamma_{\ell \ell^{\prime}}^{(k)}+\sum_{\ell^{\prime \prime} \in \mathcal{L} \backslash\left\{\ell, \ell^{\prime}\right\}} \gamma_{\ell \ell^{\prime} \ell^{\prime \prime}}^{(k)}+\ldots+\gamma_{12 \cdots L}^{(k)}\right) \leq \epsilon, \\
a_{n \ell}^{+}\left|a_{n \ell^{\prime}}^{-}\right|\left(\gamma_{\ell \ell^{\prime}}^{(k)}+\sum_{\ell^{\prime \prime} \in \mathcal{L} \backslash\left\{\ell, \ell^{\prime}\right\}} \gamma_{\ell \ell^{\prime} \ell^{\prime \prime}}^{(k)}+\ldots+\gamma_{12 \cdots L}^{(k)}\right) \leq \epsilon,
\end{gathered}
$$

where $\epsilon$ is an arbitrary small positive number. The constraints in (18) can be readily incorporated in the GP framework.

Now, the only remaining constraints that are not GPcompatible are those in (11). Invoking the change of variables in (14), we can rewrite this set of constraints for each link $\ell \in \mathcal{L}$ and subcarrier $k \in \mathcal{K}$ as follows:

$$
\begin{aligned}
\prod_{d \in \mathcal{D}} r_{\ell k}^{(d)} \leq & \left(1+p_{\ell k} g_{\ell k}\right)^{\gamma_{\ell}^{(k)}} \\
& \times \prod_{\ell^{\prime} \in \mathcal{L} \backslash\{\ell\}}\left(1+\frac{p_{\ell k} g_{\ell k}}{1+p_{\ell^{\prime} k} g_{\ell^{\prime \prime} k}}\right)^{\gamma_{\ell \ell^{\prime}}^{(k)}} \\
& \vdots \\
& \times\left(1+\frac{p_{\ell k} g_{\ell k}}{1+\sum_{\ell^{\prime}} p_{\ell^{\prime} k} g_{\ell^{\prime \prime} k}}\right)^{\gamma_{12 \cdots L}^{(k)}} .
\end{aligned}
$$

Although the LHS of (19) is in the form of a monomial, its RHS is not a monomial. However, this RHS is amenable to a GP-based approximation technique known as monomial approximation [13]. Let $z \in \mathbb{R}^{n}$ be a vector of positive entries 
and $f(z)$ be a positive differentiable function. A monomial approximation of $f(z)$ near $z_{0}, \mathcal{M}(f(z))$, is given by

$$
\mathcal{M}(f(z))=f\left(z_{0}\right) \prod_{i=1}^{n}\left(\frac{z_{i}}{z_{i 0}}\right)^{\beta_{i}},
$$

where $\beta_{i}=\left.\frac{z_{i 0}}{f\left(z_{0}\right)} \frac{\partial f}{\partial z_{i}}\right|_{z=z_{0}}$. To use this approach, one can approximate the whole RHS of (19) by one monomial. However, this approach is overly complicated, and an alternative is to approximate each term in the RHS of (19) by a monomial. Noting that the product of monomials is a monomial, it can be verified that, for each link $\ell \in \mathcal{L}$ and subcarrier $k \in \mathcal{K}$, a monomial approximation of the constraints in (11) is given by

$$
\begin{aligned}
\prod_{d \in \mathcal{D}} r_{\ell k}^{(d)} \leq & \mathcal{M}\left(\left(1+p_{\ell k} g_{\ell k}\right)^{\gamma_{\ell}^{(k)}}\right) \\
& \times \prod_{\ell^{\prime} \in \mathcal{L} \backslash\{\ell\}} \mathcal{M}\left(\left(1+\frac{p_{\ell k} g_{\ell k}}{1+p_{\ell^{\prime} k} g_{\ell^{\prime \prime k}}}\right)^{\gamma_{\ell \ell^{\prime}}^{(k)}}\right) \\
& \vdots \\
& \times \mathcal{M}\left(\left(1+\frac{p_{\ell k} g_{\ell k}}{1+\sum_{\ell^{\prime}} p_{\ell^{\prime} k} g_{\ell^{\prime \prime k}}}\right)^{\gamma_{12 \cdots L}^{(k)}}\right) .
\end{aligned}
$$

Now, the problem in (12) can be approximated by

$$
\begin{aligned}
\max _{\left\{t_{n}^{(d)}\right\},\left\{r_{\ell k}^{(d)}\right\},\left\{p_{\ell k}\right\}, \Gamma} & \prod_{d \in \mathcal{D}} \prod_{n \in \mathcal{N} \backslash\{d\}}\left(t_{n}^{(d)}\right)^{w_{n}^{(d)}}, \\
\text { subject to } & \text { Routing consts. in (15)-(17), } \\
& \text { Power allocation consts. in (10), } \\
& \text { Scheduling consts. in (6) and (18), } \\
& \text { Approx. capacity consts. in (20). }
\end{aligned}
$$

Using a standard exponential transformation, the GP in (21) can be readily transformed into a convex optimization problem which can be solved efficiently using interior point methods [13]. Although finding the global solution of the problem in (12) is difficult, finding the solution of its approximated one in (21) is straightforward. To exploit this advantage, we use this approximation in an iterative manner. More specifically, starting from a feasible point, $\left(\left\{p_{\ell k}^{(0)}\right\}, \Gamma^{(0)}\right)$, we solve the GP problem in (21). The solution is then used as an initial point for the next iteration. This method is known as the "single condensation method" and, under relatively mild conditions, its convergence to a point that satisfies the KKT conditions of the problem in (12) is guaranteed [14]. Since the problem in (12) is nonconvex, its corresponding KKT conditions are only necessary, but not sufficient, for optimality and hence, the approximated solution obtained through the iterative algorithm yields a feasible lower bound on the optimal solution.

\section{Numerical Results}

We consider an exemplary network in Figure 2 with $N=4$ nodes, $L=12$ links and $K=4$ unit bandwidth subcarriers. The nodes are randomly dropped in a $500 \times 500 \mathrm{~m}^{2}$ square and are assumed to have identical power budgets, i.e., $P_{n}=$ $P, n=1, \ldots, 4$ which is normalized to $0 \mathrm{dBm}$. The noise
TABLE I: Power allocations and time-sharing schedules generated by the proposed iterative algorithm.

\begin{tabular}{|l|l|l|l|}
\hline \multicolumn{4}{|c|}{ Power Allocations (mW) } \\
\hline$n=1$ & $n=2$ & $n=3$ & $n=4$ \\
\hline$p_{2,1}=0.32$ & $p_{5,2}=1$ & $p_{7,2}=0.55$ & $p_{11,1}=1$ \\
$p_{1,3}=0.45$ & & $p_{8,4}=0.45$ & \\
$p_{3,4}=0.23$ & & & \\
\hline \multicolumn{3}{|c|}{ Time-Sharing Subcarrier Schedules (\%) } \\
\hline$k=1$ & $k=2$ & $k=3$ & $k=4$ \\
\hline$\gamma_{2,11}=100$ & $\gamma_{3,5}=15$ & $\gamma_{1}=13$ & $\gamma_{3,8}=100$ \\
& $\gamma_{4,7}=20$ & $\gamma_{1,8}=22$ & \\
& $\gamma_{5,10}=15$ & $\gamma_{1,9}=30$ & \\
& $\gamma_{5,12}=10$ & $\gamma_{1,11}=10$ & \\
& $\gamma_{6,7}=25$ & $\gamma_{1,12}=10$ & \\
& $\gamma_{2,5,12}=15$ & $\gamma_{1,8,11}=15$ & \\
\hline
\end{tabular}

power at the receivers is $-100 \mathrm{dBm}$. Nodes 1 and 2 wish to communicate with each other. Hence, $\mathcal{D}=\{1,2\}$.

The channels are standard quasi-static frequency flat Rayleigh fading with log-normal shadowing and pathloss component. Using the modified Hata urban propagation model [3], the pathloss component is

$$
\begin{cases}8+38 \log \left(d_{\ell}\right) & \text { if } d_{\ell} \geq 50 \mathrm{~m} \\ 8+38 \log (50) & \text { if } d_{\ell}<50 \mathrm{~m}\end{cases}
$$

where $d_{\ell}$ is the length of link $\ell$ in meters. The shadowing component is assumed to have a log-normal distribution with mean of $0 \mathrm{~dB}$ and standard deviation of $\sigma_{s}=8 \mathrm{~dB}$.

Assuming equal weights, $w_{1}^{(2)}=w_{2}^{(1)}=1$, the optimization problem in (20) yields a sum-rate of 14.45 bits-per-secondper-Hertz (bps/Hz). The injected rates and the flow of active links are depicted in Figure 2. As shown in this figure, the flows determine the routes taken by each data stream. Power allocation at each node and the time-sharing schedules for each subcarrier are presented in Table I. It is worth noting that, even for a small network like the one considered in this example, the number of optimization variables is relatively large. For instance, for the considered network the number of variables is 16526 , of which 16380 are time-share variables, 98 are flow variables and 48 are power variables. Hence, one can see that the framework proposed herein is only suitable for designing small to medium size networks. For larger networks, this framework can be seen as a benchmark and a first step towards developing effective distributed designs.

By applying the GP-based algorithm in Section III for all $w_{2}^{(1)}$ and $w_{1}^{(2)}$ in the unit simplex, $\left\{\left(w_{2}^{(1)}, w_{1}^{(2)}\right) \mid w_{2}^{(1)} \geq\right.$ $\left.0, w_{1}^{(2)} \geq 0, w_{2}^{(1)}+w_{1}^{(2)}=1\right\}$, we obtain the region of all rates tuples $\left(s_{2}^{(1)}, s_{1}^{(2)}\right)$ that can be reliably communicated over the network with the algorithm in Section III. This region is depicted in Figure 3 and is compared with the regions obtained through cross-layer designs without frequency-reuse in [6]. From this figure, it can be seen that, despite its potential suboptimality, the rate region corresponding to (20) properly contains the other ones.

In Figure 4, the sum-rate yielded by the proposed optimization in (20) is compared with the sum-rate yielded by a binary and the time-shared scheduling, both without frequency-reuse. As shown in this figure, the proposed algorithm has superior 


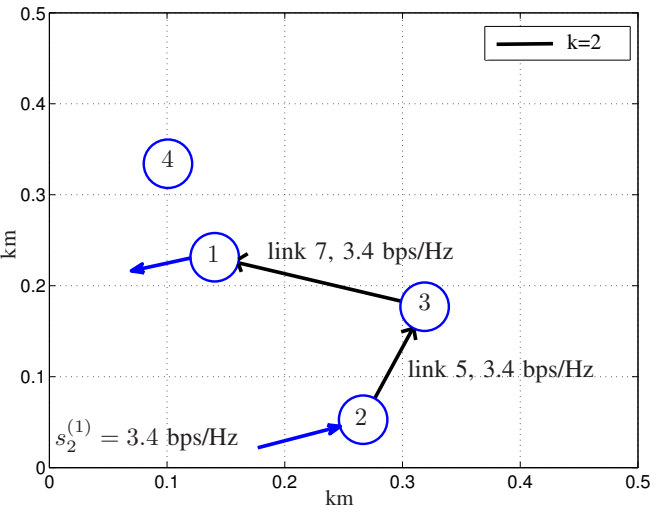

(a)

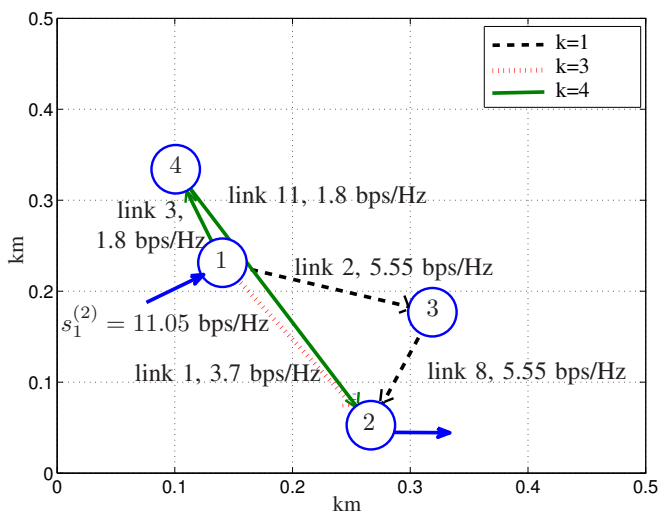

(b)

Fig. 2: Data routes generated by the proposed iterative algorithm for (a) $d=1$ (h) $d=$ ?

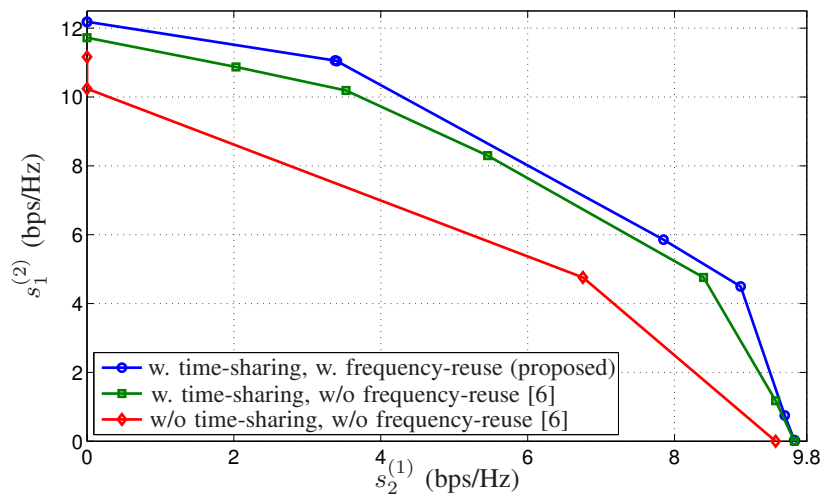

Fig. 3: Rate-region comparison.

performance especially at high power budgets.

\section{CONCLUSiON}

In this paper, we developed a framework for optimizing data routes, subcarrier schedules and power allocations in generic half-duplex interference-limited multicarrier networks. This framework subsumes designs in which time-sharing and frequency-reuse are considered separately. Considering both time-sharing and frequency-reuse jointly gives rise to generally difficult to solve non-convex optimization problems. To circumvent this difficulty, we invoked an approximation

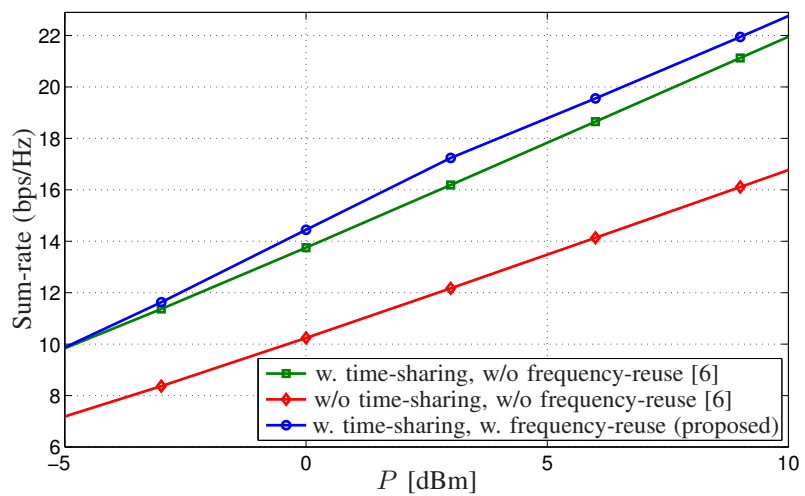

Fig. 4: Sum-rate comparison.

technique based on geometric programming and provided a computationally-efficient method for obtaining locally optimal solutions. Despite its potential performance gains, the proposed optimization framework is centralized and is thus only suitable for designing small to medium size networks. For larger networks, this framework can be seen as a benchmark and a first step towards developing effective distributed designs that make judicious use of the degrees of design freedom offered by the physical wireless medium.

\section{REFERENCES}

[1] L. Xiao, M. Johansson, and S. P. Boyd, "Simultaneous routing and resource allocation via dual decomposition," IEEE Trans. Commun., vol. 52, pp. 1136-1144, July 2004.

[2] S. Hayashi and Z.-Q. Luo, "Spectrum management for interferencelimited multiuser communication systems," IEEE Trans. Inf. Theory, vol. 55, pp. 1153-1175, Mar. 2009.

[3] K. Kim, Y. Han, and S.-L. Kim, "Joint subcarrier and power allocation in uplink OFDMA systems," IEEE Commun. Lett., vol. 9, pp. 526-528, June 2005.

[4] C. Y. Ng and C. W. Sung, "Low complexity subcarrier and power allocation for utility maximization in uplink OFDMA systems," IEEE Trans. Wireless Commun., vol. 7, pp. 1667-1675, May 2008.

[5] Y. Huang, R. H. Gohary, and Z.-Q. Luo, "Approaching user capacity in a DSL system via harmonic mean-rate optimization," in Proc. IEEE Int. Conf. Acoustics, Speech, Signal Processing, pp. 2365-2368, Apr. 2009.

[6] R. Rashtchi, R. H. Gohary, and H. Yanikomeroglu, "Routing, scheduling and power allocation in generic OFDMA wireless networks: Optimal design and efficiently computable bounds," IEEE Trans. Wireless Commun., vol. 13, pp. 2034-2046, Apr. 2014.

[7] C. Y. Wong, R. S. Cheng, K. B. Lataief, and R. D. Murch, "Multiuser OFDM with adaptive subcarrier, bit, and power allocation," IEEE J. Select. Areas Commun., vol. 17, pp. 1747-1758, Oct. 1999.

[8] J. Huang, V. G. Subramanian, R. Agrawal, and R. A. Berry, "Downlink scheduling and resource allocation for OFDM systems," IEEE Trans. Wireless Commun., vol. 8, pp. 288-296, Jan. 2009.

[9] H. Inaltekin and S. V. Hanly, "Optimality of binary power control for the single cell uplink," IEEE Trans. Inf. Theory, vol. 58, pp. 6484-6498, Oct. 2012.

[10] M. Chiang, C. W. Tan, D. P. Palomar, D. O’Neil, and D. Julian, "Power control by geometric programming," IEEE Trans. Wireless Commun., vol. 6, pp. 2640-2650, July 2007.

[11] R. Rashtchi, R. H. Gohary, and H. Yanikomeroglu, "An efficient cross layer design in OFDMA-Based wireless networks with channel reuse," in Proc. IEEE Glob. Commun. Conf. (GLOBECOM), Dec. 2013.

[12] Int. Telecommun. Union (ITU), Guidelines for Evaluation of Radio Interface Technologies for IMT-Advanced. ITU-R: TR M.21351, Dec. 2009. Available at: http://www.itu.int/pub/R-REP-M.2135-1-2009.

[13] S. Boyd, S.-J. Kim, L. Vandenberghe, and A. Hassibi, "A tutorial on geometric programming," Optimization and Engineering, vol. 8, pp. 67127, Mar. 2007.

[14] B. R. Marks and G. P. Wright, "A general inner approximation algorithm for nonconvex mathematical programs," Operations Research, vol. 26, pp. 681-683, Aug. 1978. 